\title{
Automatic diagnostic device with measurement of distances to damages by the combined pulse- phase method
}

\author{
Janusz Musiał ${ }^{1,}$ Kostyantin Horiashchenko ${ }^{2 *}$, Serhiy Horiashchenko ${ }^{2}$, Mikołaj Szyca ${ }^{1}$ \\ ${ }^{1}$ Bydgoszcz Univeristy Science and Technology, Kaliskiego 7 Street, 85-786 Bydgoszcz, Poland \\ ${ }^{2}$ Khmelnitsky National University, Instytutska Street, 11, Khmelnytskyi, 29000, Ukraine
}

\begin{abstract}
The article is devoted to the study of the state of diagnosing the integrity of cables. The current state of diagnostics of problems of damage of cables is shown. Formulas for calculating several signals reflected from damage have been removed. To ensure the automation of the measurement process, based on the functional diagram of the automatic diagnostic device with measurement of distances to damage by the combined pulse-phase method, a block diagram of an automated distance meter to damage was developed, which allows measuring the investigated cable in automatic mode. This reduces the loss of time to perform measurement operations and ensure the required sequence of actions without the participation of the operator.
\end{abstract}

\section{Introduction}

Under the influence of various external and internal factors, the cable changes its internal structure - inhomogeneity. For each of the existing types of lines, different types of heterogeneity that may occur in the cable during its operation play a greater or lesser role. The basic types of damage (heterogeneity) that occur are [1, 2]:

- short circuit between the cores or between the core and the screen and breaks (socalled "final" inhomogeneity);

- change of physical parameters of cables, such as longitudinal resistance, change of capacity or resistance between cores ("partial" heterogeneity).

To solve the problem of diagnosing damage, there are a large number of devices that are based on the use of the pulse method. Distance meters based on phase shift measurement have not found wide practical application. Devices based on pulsed sounding cables have become much more widespread $[3,4,5]$. Improving their characteristics is only due to technical means: increasing the sensitivity, increasing the power of the probing pulse signal, the use of special shape signals, mathematical processing - the use of statistical processing of information (filtering, approximation, recovery), evaluation of signal parameters such as amplitude, delay relative probing signal.

Carrying out measurements for long distances becomes more difficult with increasing signal attenuation. The use of switching devices in low-frequency communication lines is limited by the following factors:

- much more signal attenuation requires an increase in the duration of the probing

${ }^{*}$ Corresponding author: tnt7@ukr.net 
signals compared to RF cables;

- the limited bandwidth of the lines in some cases significantly reduces the distance at which the measurement can be performed.

Accordingly, it can be stated that today the problem of measuring distances in lowfrequency communication lines remains relevant.

\section{Problem statement}

Phase shift measurements are one of the most accurate measurements. Issues of use, implementation and practical use of phasemetry methods for research evaluation [6] and considered in $[7,8]$.

The method of determination is based on measurements of the shift between the outputs and reflected from the damage signals. Since it is known that the phase determination occurs at the levels of tenths and hundredths of a degree $[9,10,11]$, the error of determination will also depend on the wavelength of the probing line. Distance to the damage location in the cable $l$ defined as [Błąd! Nie można odnaleźć źródła odwołania.0]:

$$
l=\frac{\lambda \cdot \varphi}{2 \cdot 2 \pi}
$$

where $\lambda$-wavelength of the probing signal;

$\varphi$ - phase shift angle.

Let's analyze the accuracy of the method. For example, as can be seen from formula (1), given the wavelength of $1 \mathrm{~m}$ (corresponding to a frequency of $300 \mathrm{MHz}$ ) and the error of determining the phase shift of $0.1 \%\left(0.36^{\circ}\right)$, the error of determining the distance will be only:

$$
\Delta l=\frac{1 \cdot(2 \pi \cdot 0,1 \%)}{2 \cdot 2 \pi}=5 \cdot 10^{-4} \mathrm{~m}
$$

One of the problems when using this method is the case of uncertainty of distance measurement, which occurs when the wavelength is less than the cable length. $L$.

Then $l=\frac{\lambda(2 \pi \cdot n+\varphi)}{2 \pi}$, where $n-$ integer from 0 to $[L / \lambda]$. In this case, the actual value of the phase shift angle $\varphi-$ unknown.

A number of methods that can eliminate these uncertainties. For example, for systems with high frequency signals (satellite positioning system, GPS) to increase the accuracy of determining the distance to one object using the method of improving the accuracy at a known base distance - the so-called "combined wave detection" [12], which is based on using two signals of different frequencies $\lambda_{1}$ and $\lambda_{2}$.

To accurately measure the unknown distance, the so-called multi-scale method is used $[6,11]$. The formation of a number of frequencies from the lowest, the wavelength of which is necessarily not less than the length of the cable (which eliminates the ambiguity of the transition through $2 \pi$ ) to the highest, the period of which will determine the smallest error in determining the distance.

We highlight the following advantages of the method of measuring phase shift:

- High accuracy of determination of phase shift allows receiving high accuracy of definition of distance to damage; 
- The use of low frequencies during measurements can greatly simplify the construction of the meter, reduces the sensitivity to external factors;

- Phase determination is possible for significant time intervals, which significantly improves accuracy.

\section{Analysis of recent references and publications}

Currently known methods for determining damage in communication lines [13, 14,15, 16], which are based on measuring various physical parameters of lines (resistance) or measuring signals (time of reflection or phase shift between probing and reflected signals).

According to the measurement time, the methods of measuring distances can be divided into two groups:

1) Measurement of dynamic parameters (measurement of values that change constantly over time);

2) Measurement of static parameters (measurement of values, the values of which are constant throughout the measurement time).

It should be noted that the value of the measured value can be considered constant (unchanged) during the measurement time, if the limits of change of the value of this value are less than the sensitivity of the measuring device. Consider the advantages and disadvantages of several basic methods for determining the distances to the damage. In part, other authors performed such an analysis in $[17,18,19,20,21]$.

In general, the measurement process can be divided into 3 successive stages: 1 st and 2 nd - measurement, 3rd - calculation. In more detail, these steps can be written as follows:

1 st stage. Determining the number of injuries $k$, their nature and approximate distance $l_{k}$ to each of the damage. This problem is solved with the help of a pulse reflectometer, which performs the following actions: A pulse signal of a given duration is formed $\Delta t$ and amplitude $U_{O}$, which is fed into the line under study.

When reflecting the probing signal from objects in the line, we obtain a model of the reflected signal, which can be represented as:

$$
S_{B}=\left(U_{0} \cdot e^{-\beta \cdot l}\right) \cdot K \cdot e^{-\beta \cdot l} \cdot \cos (\omega \cdot t)=K \cdot U_{0} \cdot e^{-\beta \cdot 2 l} \cdot \cos (\omega \cdot t)
$$

where $K$ - damage reflection coefficient;

$l$ - the distance from the damage to the signal source, or from the first damage to the second.

The signal is measured from the cables. Each reflection determines the change in amplitude in the received signal. At the same time the time of appearance of reflection is fixed $t_{k}$ and signal amplitude $U_{k}$.

2nd stage. Determination of the phase shift angle between the probing and reflected signals. As already shown in Section 2, to solve equation that necessary to measure the phase shift between the probe and the reflected total signal at different frequencies.

Because of the existence of signals from each damage simultaneously and their interference, we obtain a total signal of the form:

$$
\begin{aligned}
& s_{x}(t)=s_{x 1}(t)+s_{x 2}(t)+\ldots+s_{x k}(t)= \\
& =U_{1} \cos \left(\omega t+\varphi_{1}\right)+U_{2} \cos \left(\omega t+\varphi_{2}\right)+\ldots+U_{k} \cos \left(\omega t+\varphi_{k}\right)= \\
& =\sum_{k=1}^{K} U_{k} \cos \left(\omega t+\varphi_{k}\right)
\end{aligned}
$$


where $\quad k$-number of reflections.

The phase shift measurement is performed by a phase meter. At this stage, the following steps must be performed: At the output of the phase shift meter, a continuous sinusoidal signal with a known frequency is formed $f_{i}$, starting from the smallest value determined from formula. At the input, we obtain a signal of the form. From the received signal is subtracted the known signal, which is generated by the generator harmonic phase shifter. The total phase shift angle is determined $\varphi_{\Sigma}$ and from the known times of the appearance of the reflections $t_{k}$ using calculate the phase shift angles $\varphi_{k}$, formed by each damage $t_{k}$.

The measurement cycle is repeated for a number of known frequencies.

3rd stage. Solution of the system of equations (3).

The values of the phase shifts in the low-frequency line practically depend only on the distances to the objects. By repeating all the above mathematical calculations for each frequency for the system, you can get the following system of equations [23, 24]:

$$
\left\{\begin{array}{l}
\sum_{k=1}^{K} U_{\kappa} \cos \left(\varphi_{1, k}\right)=A_{\Sigma 1} \cos \varphi_{\Sigma 1} \\
\sum_{k=1}^{K} U_{\kappa} \cos \left(\varphi_{2, k}\right)=A_{\Sigma 2} \cos \varphi_{\Sigma 2} \\
\ldots \ldots \ldots \ldots \ldots \ldots \ldots \ldots \ldots \ldots \ldots \ldots \\
\sum_{k=1}^{K} U_{\kappa} \cos \left(\varphi_{N, k}\right)=A_{\Sigma N} \cos \varphi_{\Sigma N}
\end{array}\right.
$$

where $\varphi_{N, k}$ - phase shift angle for -th damage to the $N$-th probing signal;

$A_{\Sigma N}$ - the total amplitude of the reflected signal for $N$-th probing signal;

$\varphi_{\Sigma N}$ - total phase shift angle for $N$-th probing signal;

$U_{\kappa}$ - he amplitude of the reflected signal $k$-th damage.

The total number of equations $N$ is determined based on the number of damages. In the case of 2 damages, the equation of system is reduced to the next system of two equations.

\section{Presenting main material}

Based on the above stages of measurement, a general block diagram of the installation for research of the pulse-phase method of determining the distances to damage in the conductive communication lines, based on which further development of the measuring device is possible.

An example of a functional diagram of a device that implements the considered block diagram, which is used to determine the length of the electric transmission cable to the damaged places, is shown in Fig. 1.

The device consists of the following components: clock generator 1 (GTS), control and calculation device 2 (CCD), which also contains means of data exchange and indication, pulse generator 3 (GI), sinusoidal signal generator 4 (GSS), analog-digital converter 5 (ADC), signal switch 6 (CS), matching and separation device 7 (MDS). In addition, the test line 8 and the agreed load 9 are connected to the circuit.

GI 3 and ADC 5 are used to implement the IP unit (Fig. 1). GSS and ADC 5 are used to operate the VF unit. Data from ADC 5 is sent to CCD 2, which performs operations on 
recording and further processing of information. The device of coordination of PU is realized by means of KS 6 and MDS 7.

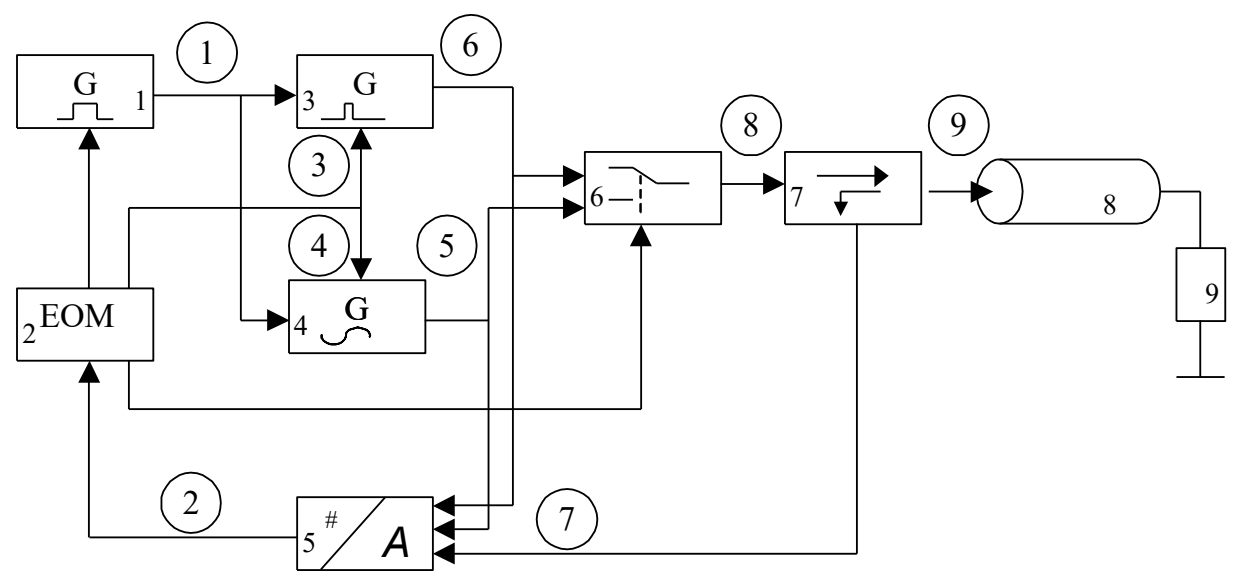

Fig.1. Functional diagram of a device for measuring distance pulse-phase method:

1 - clock generator (GTS);

2 - control and calculation device (CCD);

3 - impulse generator (GI);

4 - generator of sinusoidal signals (GSS);

5 - analog-to-digital converter (ADC);

6- signal switch (CS);

7 - matching device and separation (MDS);

8 - the studied line;

9 - agreed load.

Consider in more detail the functional diagram of the device (Fig.1). GTS 1 generates clock signals for the operation of GI 3 and GSS 4. The output of GTS 1 is connected to GI 2 and GSS 4 . The outputs of generators 2 and 4 are connected to GS 6, the output of which is connected to the input MDS 7.

The output of the MDS 7 is connected to the test cable 8, the other end of which is loaded on the agreed load 9. The output of the MDS 7, GI 3 and GSS 4 is connected to the ADC 5. CCD 2 is connected to the GTS 1, GI 3, GSS 4, COP 6 and ADC 5. Diagrams of the presented scheme are shown in Fig.2.

Mathematical processing of the obtained values. The final processing of the measurement results is performed using CCD 2. Using the data obtained in steps 1 and 2 for the system of equations (4):

$$
\left\{\begin{array}{l}
\sum_{k=1}^{2} U_{k} \cos \left(\varphi_{1, k}\right)=\frac{A_{\Sigma 1}}{\sqrt{1+\operatorname{tg}^{2} \varphi_{\Sigma 1}}} \\
\sum_{k=1}^{2} U_{k} \cos \left(\varphi_{2, k}\right)=\frac{A_{\Sigma 2}}{\sqrt{1+\operatorname{tg}^{2} \varphi_{\Sigma 2}}}
\end{array}\right.
$$




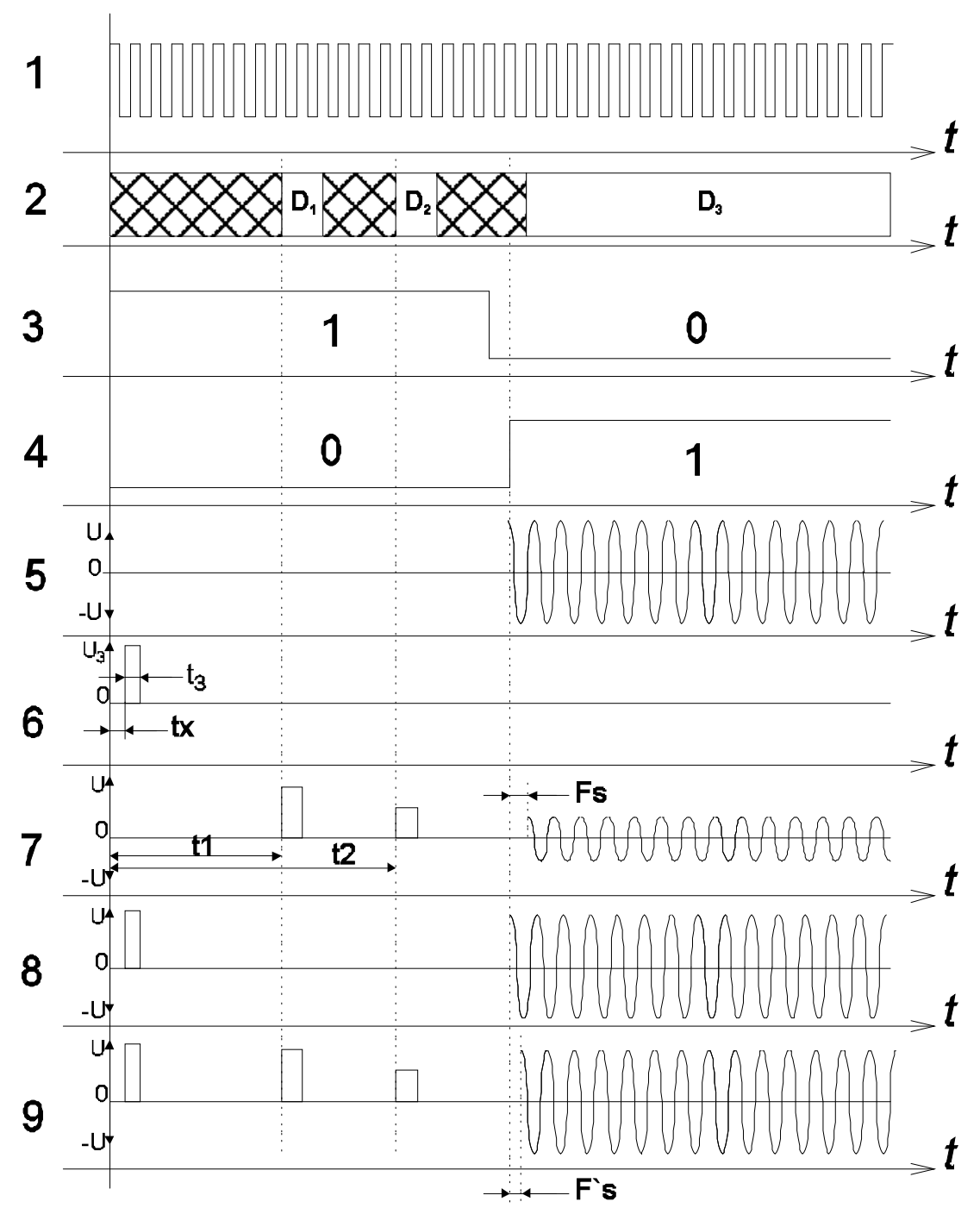

Fig.2. Diagrams of operation of the meter of distances to damages.

The calculation of the specified distances to damages is carried out. To do this, we use the algorithm of the iterative solution considered in Section 2 (Fig.3), which is implemented using the proposed software.

To perform the previously discussed 3 stages of measurement, it is necessary to develop a device that uses the proposed block diagram of the distance meter to damage.

For development of the scheme of the automated measuring instrument of distances to damages we will take as a basis the functional scheme considered earlier (Fig.2). The use of modern automation tools due to the transition from hardware to hardware and software implementation can further reduce the complexity of the scheme.

The use of software and hardware implementation makes it possible to transfer part of the functions of the circuit to the device control program $m$ and make the meter not inherent in the measuring instruments of previous years capabilities $[25,26,27$,$] . Such as: processing$ the input signal to select a useful signal; saving data results for further processing; formation of source information in the form of figures and tables; condition change control cables [28, 
29, 30].

The CCD unit performs actions to set the operating modes of the nodes. We implement it on the basis of a computer. The exchange of data between the computer and the nodes is performed using control registers 3-7. Control registers 3-7 are used to set the appropriate operating modes of the circuit. Thus, for hardware implementation it remains to develop signal generators (GI and GSS), ADC, signal switch and matching device.

The developed block diagram of the automatic diagnostic device with measurement of distances to damage by the combined pulse-phase method is shown in Fig.3.

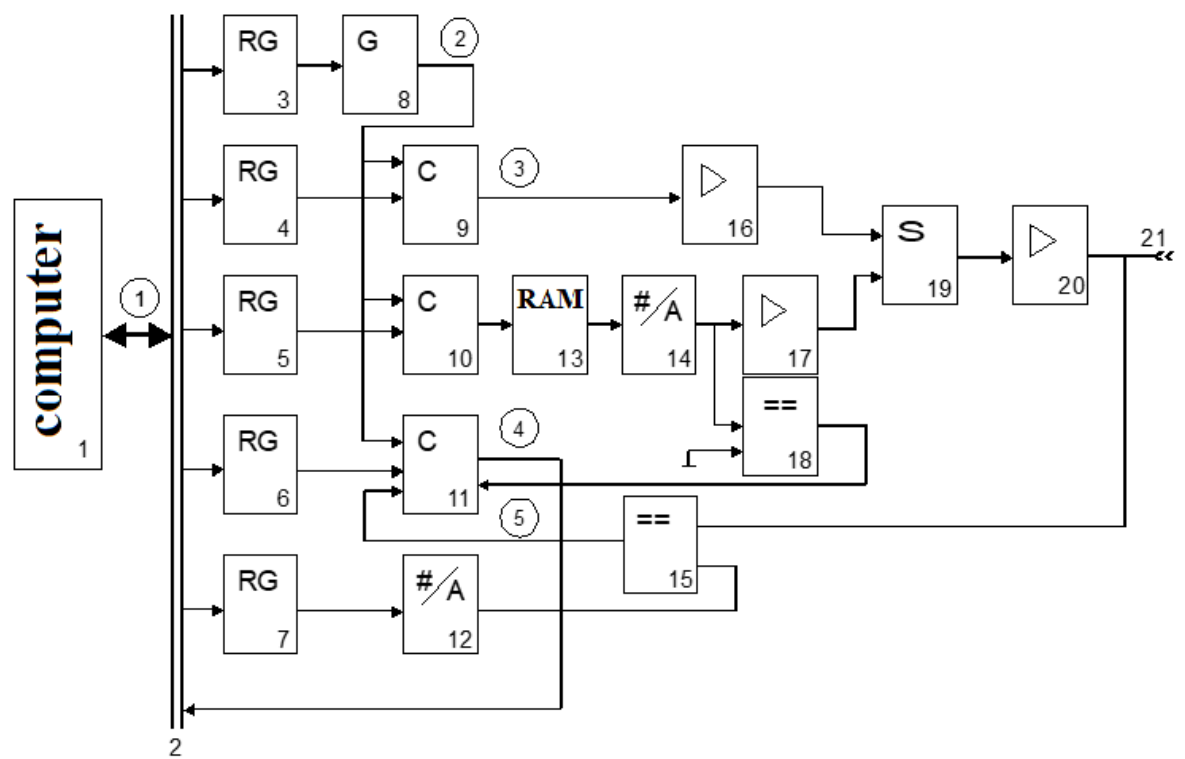

Fig.3. Block diagram of an automatic diagnostic device with measurement of distances to damage by the combined pulse-phase method.

The block diagram of the meter consists of the following blocks: The computer 1 through the data bus and control (SDC) 2 is combined with the control registers 3, 4, 5, 6, 7 and the output of the clock counter (LTS) 11. The output of the register 3 is connected to the input controlled clock generator (GTS) 8. The output of the register 4 is connected to the input of the counter 9 - the pulse generator (GI). The output of register 5 is connected to the input of the address counter (LA) 10. The output of register 6 is connected to the input of LTS 11. The output of register 7 is connected to the input of DAC 12. The output of GTS 8 is connected to inputs of counters 9,10 and 11 . The output of the counter 9 is connected to the input of the amplifier 16. The output of the counter 10 is connected to the input of the ROM 13. The output of the ROM 13 is connected to the input of the DAC 14, the output of which is connected to the input of the amplifier 17 and the comparator start signal 18. Signals from amplifiers 16 and 17 are fed to the adder 19, the output of which is connected to the output amplifier 20. The output of the amplifier 20 is directly connected to the output of the device 21 and simultaneously to the input of the comparator 15 . The output of the DAC 12 is connected to the second input comparator 15 , the output of which is connected to the input of the meter. The output of the comparator 18 is connected to the meter 11 .

The operation of the device is controlled by a computer 1, the task of which is to transmit the necessary data (codes of clock frequency, harmonic signal frequency, pulse duration) to the control registers and read the obtained values (reflection time, signal amplitude values).

The work process begins with loading into register 3 the code of the required clock frequency. According to the selected measurement mode, register 4 or 5 is selected. 
Thus, the register 4 and the counter 9 perform the function of a pulse generator for operation in the mode of the pulse reflectometer. The register 4 is designed to store a code of the required pulse duration, based on which the counter 9 will generate a signal of a certain duration. According to the signal "clock" from the generator 8 , the counter 9 from the register 4 is loaded with the pulse duration code.

In the mode of the phase-shift angle meter, the value of the increment step of the values of the counter 10 is recorded in the register 5 . The counter 10 performs sequential address search in the ROM 13 with the specified step value. The ROM 13 is designed to store the harmonic signal translated into digital code. The digital code from the ROM 13 is fed to the DAC 14, which generates a corresponding output analog signal. By changing the increment step in register 5, the frequency of the signal at the output of the DAC changes.

The pulse signal from the output of the counter 9 goes through the amplifier 16 . The harmonic signal from the DAC 14 is fed to the amplifier 17. Pulse and harmonic signals are fed to the inputs of the adder 19, from which the signal goes to the output amplifier 20 with variable output resistance.

To measure the value of the amplitude of the input signal uses a gated ADC, which is implemented using registers 6 and 7, the counter 11, the DAC 12 and the comparator 15. Time diagrams of ADC operation are shown in Fig.4. Computer 1 sequentially loads the pulse duration code or the clock frequency code of the probe signal synthesizer, the clock frequency code (code f, pos. 1), the DAC output voltage value code. The next step is to start the measuring system. The computer puts the SDC in read mode and waits for the count to complete. After that, the time code is read from the counter 11.

The counter 11 in its work uses data from registers 6 and 7 and the comparator 15. The register 6 stores the delay time code, during which, starting from the beginning of the count, the counter 11 does not respond to signals from the comparator 15.

In the register 7 is loaded the code of the output voltage of the DAC 12. After the specified time, the counter 11 stops the digit only after the signal from the comparator 15 or when the counter is overflowing.

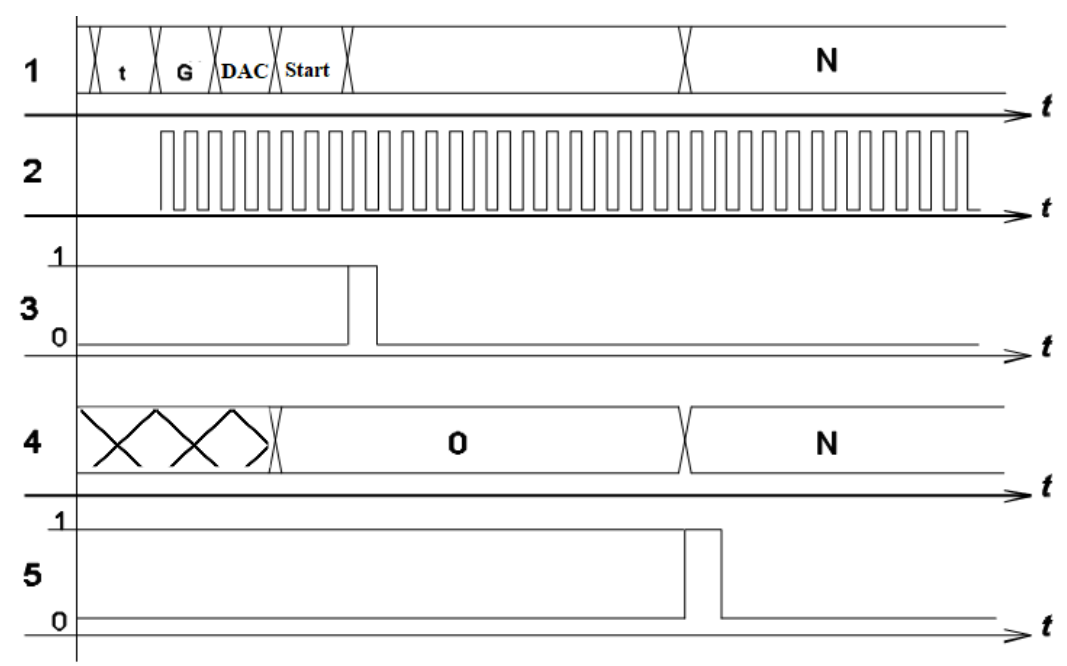

Fig.4. Timing diagrams of the automatic diagnostic device with measurement of distances to damage by the combined pulse-phase method. Pulse meter operation mode. 1 - Control data; 2 - Clock signal; 3 - pulse probing signal; 4 - operation of the meter; 5 - input pulse from the comparator.

The process of measuring the level of the output signal with the performed method of sequential approximation was experimentally tested. The general appearance of the stand is 


\section{shown in Fig.5.}

If the level of the input signal is greater than (or equal to) the set, the counter 11 will stop at the same time that was set in the register 6 . If the amplitude of the input signal is less than the set threshold, the comparator 15 will not generate a signal (pos. 4, fig. 5). In this case, the time value code obtained at the output of the counter 11 will be much larger than the set time in accordance with (4) in the register 6.

The developed distance meter will make its errors in the process of measuring values. Its instrumental errors will be determined by the element base used.

As can be seen from the functional diagram of the device (Fig.4), The main units of the measuring system are the following nodes: output signal generators (pulse generator 5 and harmonic signal 6) and analog-to-digital converter 7. Parameters such as speed and signal impact are important for them. Therefore, in this section a more detailed analysis of the development of these nodes of the scheme.

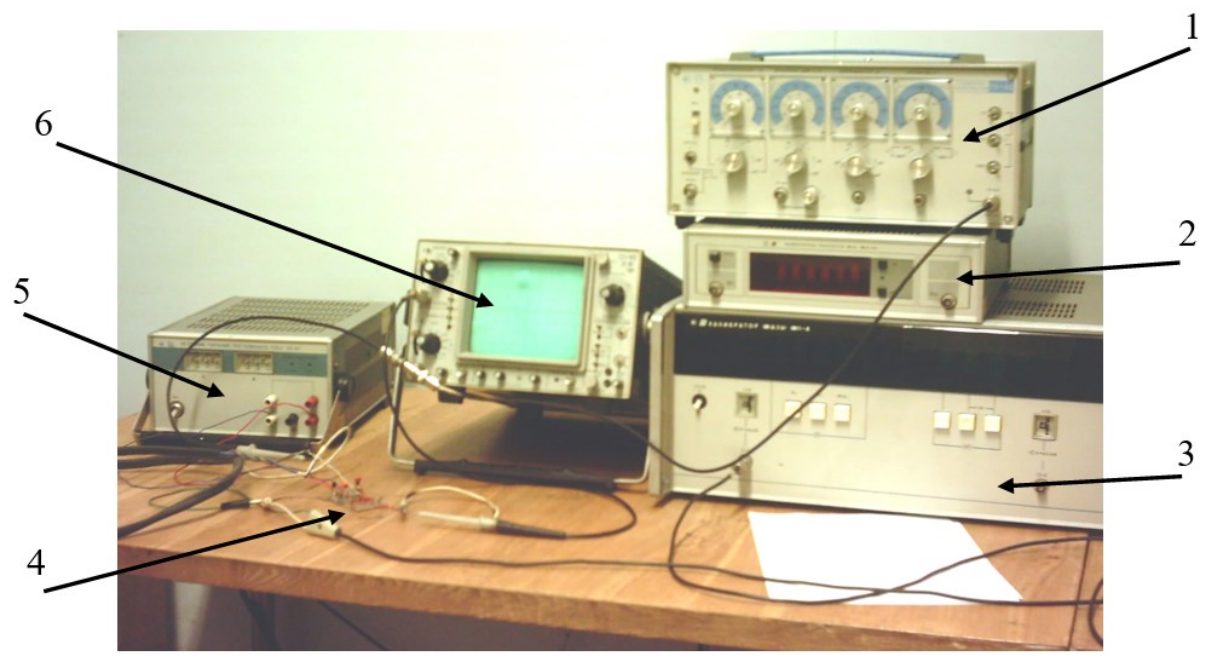

Fig.5. Experimental test of automatic diagnostic for sounding cables pulse signals: 1) pulse generator G5-63; 2) measuring the phase difference F2-34; 3) phase calibrator F1-4; 4) amplifier unit P1-P5; 5) power supply; 6) oscilloscope C1-99.

Transfer of reflections in the table at different wavelengths of the probing signal shows on Fig. 6 and Fig.7. Where $\lambda$ - probing signal; $L$ - cable length, relative to the length of the probing signal $\lambda ; X 1, X 2$ - distance to inhomogeneity; $F 1, F 2$ - phase shift angle from each inhomogeneity, radians. 

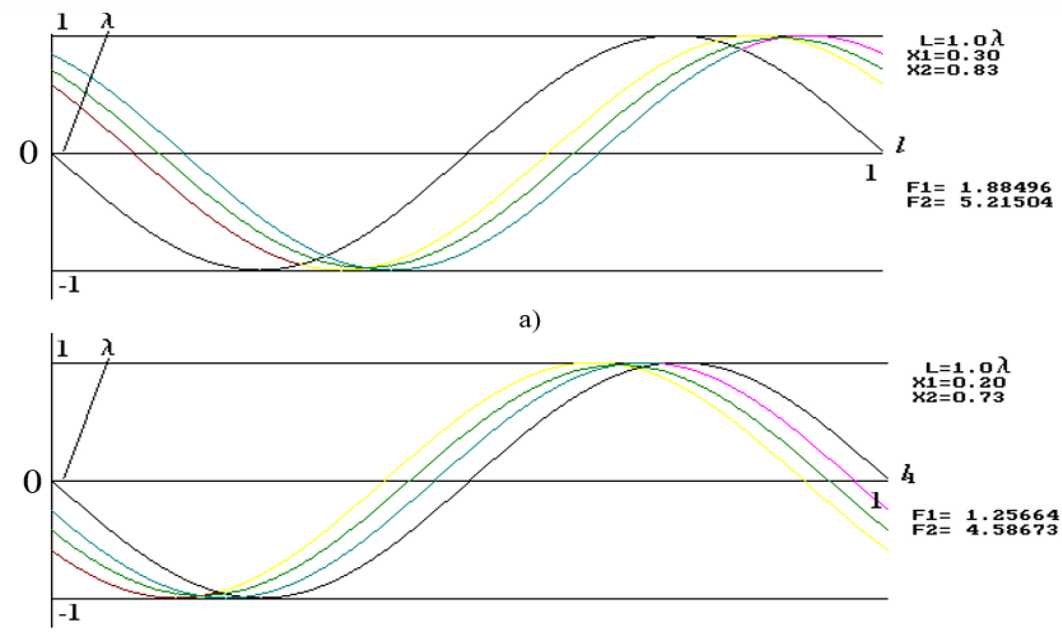

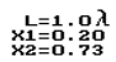

b)

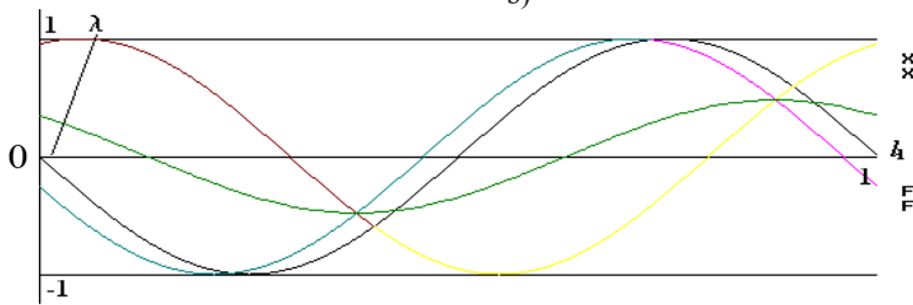

$F 1=1.25664$

Fig.6. Phase shift signals for $L=1 \cdot \lambda$.

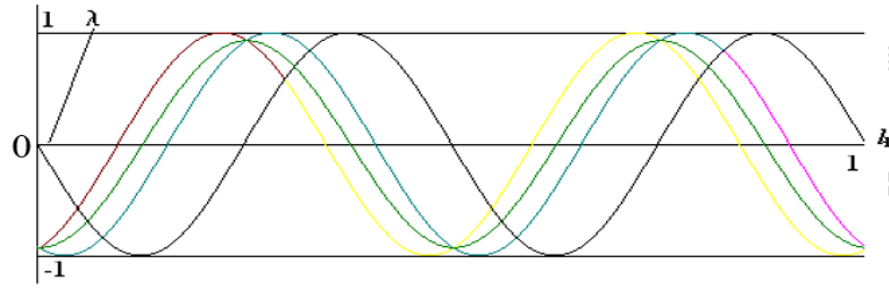

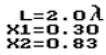

$\mathrm{F} 2=2.51327$

a)

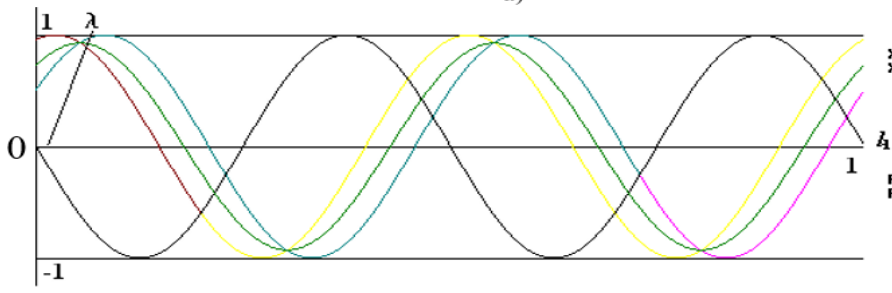

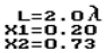

$F 1=3: 76991$

b)

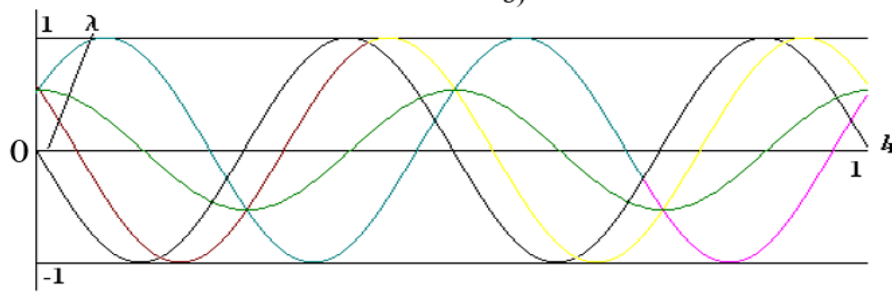

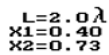

F1= $2.5132 ?$

c)

Fig.7. Phase shift signals for $L=2 \cdot \lambda$. 


\section{Conclusions}

In accordance with the analysis of the values to be determined for use in the pulse-phase measurement method, a general block diagram of a distance meter that implements this method is proposed and the sequence of stages of the measurement process is determined. The general block diagram allows you to determine the main nodes that need to be created. The functional scheme of the device for distance measurement by the pulse-phase method is developed. A sequential description of all stages of measurement is shown, for which the operation of the device in the mode of a pulse reflectometer and measurement of the phase shift angle is considered. The device operation diagrams for each of these measurement steps are given, showing the main signals of the device and the relationship between these signals over time.

\section{References}

1. C.F.Jensen (2014) Series Phase and Sequence Impedance Matrices of Crossbonded Cable Systems. In: Online Location of Faults on AC Cables in Underground Transmission Systems. Springer Theses (Recognizing Outstanding Ph.D. Research). Springer, Cham. https://doi.org/10.1007/978-3-319-05398-1_4

2. Ch.Fibre: Connection to the future. Technical overview. The Fibre Channel Association. - Published by LLH Technology Publishing, 66 (1998).

3. G. Glebowić, A. Andrianov, Y. Vvedensky et at. Research of objects by means of picosecond impulses. Radio and communication, 256 (1984).

4. V. Colloca, A. Fara, M. Nigris, G. Rizzi, Comparison among different diagnostic systems for medium voltage cable lines, paper 15-109, CIRED 2001 Paris, 5 (2001).

5. G. Hoff, H. Kranz, Correlation Between Return Voltage and Relaxation Current Measurements on XLPE Medium Voltage Cables, ISH, London, UK, paper 5.102. 14 (1999).

6. S. Ruscak, L. Singer, Using Histogram Techniques to. Measure A/D Converter Noise, An. Dial. 29-2, (1995).

7. Patent Of Ukraine 36460 A. K1. G01R31, (2001) Device for determining the place of damage to electric transmission lines/ Y. Skripnik, M. Skiruta, O. Kuznetsov //16.04.2001, бюл. № 3

8. Chi, P., Zhang, Z., Liang, R. et al. A fault diagnosis method of double-layer LSTM for $10 \mathrm{kV}$ single-core cable based on multiple observable electrical quantities. Electr Eng (2021). https://doi.org/10.1007/s00202-021-01324-3)

9. .Woodward P.M. Probability and Information Theory with Application to Radar. - New York: MeGraw-Hill Book Company, (1953).

10. Patent Of Ukraine 108172 G01S 13/00, Multi-frequency phase method of measuring the range of objects in the radio range/ Lyubchyk Vitaliy, Shinkaruk Oleh, Klepikovsky Andriy, Lantvoyt Maksym//Bul. № 6/2015 - 7 pp.

11. V. Lyubchik, Measurement of frequency characteristics of radio signals with a rectangular bypass spectrum, dis ... on zdob. Ph.D. Khmelnytsky, 174 (2001).

12. Single-phase method. In: Dictionary Geotechnical Engineering/Wörterbuch GeoTechnik. Springer, Berlin, Heidelberg. https://doi.org/10.1007/978-3-642-41714-6_193827

13. E. Gulski, J. Smit, P. Seitz, J. Smit, PD measurements on-site using oscillating wave test system, Conference Record of the 1998 IEEE International Symposium on Electrical Insulation, 7-10 June 1998, Arligton, USA (1998). 
14. F. Petzold, H. Schlapp, E. Gulski, P. Seitz, B. Quak, Advanced solution for on-site diagnosis of distribution power cables, IEEE Trans. on Diel. Ele. Ins. 15(6), 1584 (2008).

15. B. Jiang. Mobile monitoring of underground cable systems. A thesis ... for the degree of Master of Science in Electrical Engineering. University of Washington, 91 (2003).

16. W. Reder, D. Flaten, Reliability Centered Maintenance for Distribution Underground Systems, 2000 Power Engineering Society Summer Meeting, IEEE, 16-20 July 2000, Seattle, USA (2000).

17. S. Tulchynska, N. Shevchuk, O. Popelo, A. Pohrebniak, Y. Kravchyk, Operation of industrial parks in the conditions of sustainable development and the paradigm of circular economy. Laplage em Revista (International) 7(3C), 238-247 (2021).

18. Lal, A., Kedia, T. \& Verma, A. Underground Cable Fault Distance Locator and Counter Based Damaged Cable Replacement Indicator System. J. Inst. Eng. India Ser. B 101, 299-308 (2020). https://doi.org/10.1007/s40031-020-00453-w.

19. Jean Carlos Hernández Mejía. Characterization of real power cable defects by diagnostic measurements. Degree PhD in the School of Electrical and Computer Engineering. Georgia Institute of Technology, 292 (2008).

20. R. Hartlein, N. Hampton, J. Hernández, J. Perkel, Overview of cable system diagnostic technologies and application. The National Electric Energy Testing Research and Applications Center (NEETRAC), Cable Diagnostic Focus Initiative Project (CDFI) 04211 and 04-212, (2006).

21. V. Liubchyk, A. Kylimnik S. Horyashchenko, Application of the Multi-frequency Phase Method of Ranging to Many Objects for Construction of Ground Penetrating Radar, International Radar Symposium (IRS), 19-21 May 2013, Dresden, Germany (2013).

22. V. Liubchyk, S. Karvan, G. Paraska Model of transmission of probing signals in the study of nano-objects 12th IEEE International Conference on Nanotechnology, 20-23 Aug. 2012, Birmingham, UK (2012).

23. J. Musiał, S. Horiashchenko, K. Horiashchenko, J. Wilczarska, Diagnosis of multilayer structures and composite parts by multifrequency phase detection, 19th International Conference Diagnostics of Machines and Vehicles "Hybrid Multimedia Mobile Stage", 15-16 Dec. 2020, Bydgoszcz, Poland (2020).

24. M. Palacz, Spectral Methods for Modelling of Wave Propagation in Structures in Terms of Damage Detection-A Review Appl. Sci. 8(7), 1124

(2018). https://doi.org/10.3390/app8071124

25. K. Kumar, T. Saravanan, R. Sreekala, N. Gopalakrishnan, K. Mini, Structural damage detection through longitudinal wave propagation using spectral finite element method, Geomech. and Eng., 12(1), 161-183 (2017). https://doi.org/10.12989/gae.2017.12.1.161.

26. Horiashchenko, S.; Horiashchenko, K.; Musiał, J. Methodology of measuring spraying the droplet flow of polymers from nozzle. Mechanika 26, 82 (2020).

27. K. Horiaschenko, V. Stetcuk, Modified method of spectral analysis of the reflection signal for damage detection systems in power lines, Kaunas, JVE International Ltd (2018).

28. K. Horiaschenko, Pulse-phase measurements for a line with two inhomogeneities, Measuring and computing engineering in technological processes, Khmelnytskyi, No. 1, p. 80-82, (2003).

29. S. Horiashchenko. O. Polishchuk. M. Łukasiewicz, M. Matuszewski, Systems of vibration parameters automated control for diagnostics of equipment technical state, 19th International Conference Diagnostics of Machines and Vehicles "Hybrid Multimedia Mobile Stage", 15-16 Dec. 2020, Bydgoszcz, Poland (2020).

30. S. Horiashchenko, I. Golinka, A. Bubulis, V. Jurenas, Simulation and Research of the Nozzle with an Ultrasonic Resonator for Spraying Polymeric Materials, Mechanika, Vol. 24, No 1, p. 61-64, (2018).

31. Transmitted Wavefront [Electronic resource] 
https://www.zygo.com/applications/measurements/transmitted-wavefront

32. V.Martynyuk, O.Eromenko, J.Boiko, T.Kałaczyński. Diagnostics of supercapacitors. Matec Web of Conferences. Pieczyska (2018)

33. T.Kałaczyński, M.Łukasiewicz, J.Musiał, T.Kasprowicz. Analysis of the diagnostic potential thermovision research in the technical condition assessment of spark ignition engines injectors. Engineering Mechanics. Czech Republic (2020)

34. M.Liss, R.Zinko, O.Polishchuk, M.Łukasiewicz, G.Betancur. Principles of formation of a vehicle's braking system complex diagnostics. Matec Web of Conferences (2021)

35. B.Landowski, E.Kuliś. Impact of the epidemiological situation in Poland in 2020 on the public transport system. Matec Web of Conferences (2021) 